\title{
Optimum Elements Selection in IRS-Assisted UAV Communications with Phase Errors
}

\author{
S. Jangsher, Member, IEEE, M. Al-Jarrah, Member, IEEE, A. Al-Dweik, Senior Member, IEEE, E. Alusa, Senior \\ Member, IEEE, and M.-S. Alouini, Fellow, IEEE
}

\begin{abstract}
This letter considers minimizing the bit error rate (BER) of unmanned aerial vehicle (UAV) communications assisted by intelligent reflecting surfaces (IRSs). By noting that increasing the number of IRS elements in the presence of phase errors does not necessarily improve the BER, it is crucial to use only the elements that contribute to reducing the BER. Consequently, we propose an efficient algorithm to activate only the elements that improve the BER. The proposed algorithm has lower complexity and comparable BER to the optimum selection process, which is an NP-hard problem. The accuracy of the estimated phase is evaluated by deriving the probability distribution function (PDF) of the least-square (LS) channel estimator, and showing that the PDF can be closely approximated by the von Mises distribution at high signal-to-noise ratios (SNRs). The obtained analytical and simulation results show that using all the available reflectors can significantly deteriorate the BER, and thus, elements' selection is necessary. In particular scenarios, using about $26 \%$ of the reflectors provides more than 10 fold BER reduction.
\end{abstract}

Index Terms-Intelligent reflecting surfaces (IRS), phase estimation, imperfect phase, phase compensation, unmanned aerial vehicle (UAV), bit error rate (BER), phase error.

\section{INTRODUCTION}

Unmanned aerial vehicles (UAVs) based communications have been on a rise with the aim of providing higher transmission rates and better link reliability for wireless communications systems [1]. However, the promised reliability and transmission rates may degrade if a line-of-sight (LoS) link is not guaranteed. To ensure the auspicious reliability, intelligent reflecting surface (IRS) assisted UAVs can be a promising solution as IRS can be designed to provide high quality communications links [1]. To achieve this goal, the reflected signals from each IRS element should be added coherently by adjusting the phase of each IRS element. Most of the work in the literature demonstrates that increasing the number of reflecting elements improves the signal quality with perfect phase estimation and compensation [2], or when all reflectors have equal phase errors statistics [3], [4]. However, when the statistical properties of the phase error for reflectors are not identical, then

S. Jangsher is with the Department of Electrical Engineering and Computer Science, Khalifa University, AbuDhabi, UAE, and also with Wireless and Signal Processing Lab, Institute of Space Technology Islamabad, Pakistan, (E-mail: sobia.jangsher@ku.ac.ae, sobia.jangsher@ist.edu.pk).

M. Al-Jarrah and E. Alsusa are with the School of Electrical and Electronic Engineering, University of manchester, Manchester M13 9PL, U.K. (Email: $\{$ mohammad.al-jarrah, E.alsusa $\} @$ manchester.ac.uk).

A. Al-Dweik is with the Center for Cyber Physical Systems, Khalifa University, AbuDhabi, UAE, (Email: arafat.dweik@ku.ac.ae). Also, he is with the Department of Electrical and Computer Engineering, Western University, London, ON, Canada, (E-mail: dweik@fulbrightmail.org).

M.-S Alouini is with King Abdullah University of Science and Technology (KAUST), Thuwal, Makkah Province, Kingdom of Saudi Arabia, (Email:slim.alouini@kaust.edu.sa). increasing the number of reflecting elements does not necessarily improve the link quality.

Generally speaking, the phase estimation and compensation of IRS cascaded channels are challenging processes and can not be performed perfectly. Therefore, the impact of phase alignment on IRS performance has been considered widely in the literature [3]-[9]. For example, bit error rate (BER) and outage probability expressions have been derived in the presence of phase errors for a single user IRS under different channel models including LoS [3] and Nakagami- $m$ fading [9]. The achievable capacity for IRS assisted UAV communications under LoS assumption is analyzed in [4]. The impact of the phase quantization errors on the achievable capacity is discussed in [5] where it is shown that phase quantization and phase noise can severely degrade the achievable rate.

As can be noted from the cited literature and the references listed therein, the system performance is typically analyzed while considering that all IRS elements have the same phase error statistics. For example, Al-Jarrah et al. [2]-[4] modeled the phase error of each reflecting element as a von Mises random variable with particular concentration factor $\kappa$, but the performance is evaluated while assuming that the same $\kappa$ value is common for all reflecting elements. However, practically this is not the case since phase estimation accuracy may vary for different reflecting elements. Consequently, the contribution of each reflecting element to the overall signal quality depends on its phase estimation accuracy. In certain scenarios, it can be noted that certain elements may deteriorate the signal quality, and thus such elements should be deactivated.

To the best of the authors' knowledge, there is no work in the literature that considers the impact of the individual reflecting elements or applies elements' selection based on their contribution to BER improvement. Therefore, this letter considers optimizing the IRS elements' selection process to reduce the link BER. The phase error distribution is derived for the case of LoS communications in closed-form, and it is shown that it can be closely approximated to the von Mises distribution. Due to the complexity of the optimum selection rule, an efficient selection rule called sort-anddrop selection (SDS) is proposed to provide near-optimum results at a lower complexity. Moreover, the SDS results are compared to the Genetic algorithm (GA) [10].

\section{System ANd Channel Models}

This work considers an IRS-enabled high frequency UAV communications as shown in Fig. 11. The base station (BS) is transmitting to a low altitude UAV (LAUAV) with the assistance of an IRS panel attached to a relatively higher altitude platform (HAP). The IRS panel consists of $L$ reflecting elements $\mathcal{R}_{1}, \mathcal{R}_{2}, \ldots, \mathcal{R}_{L}$, and 


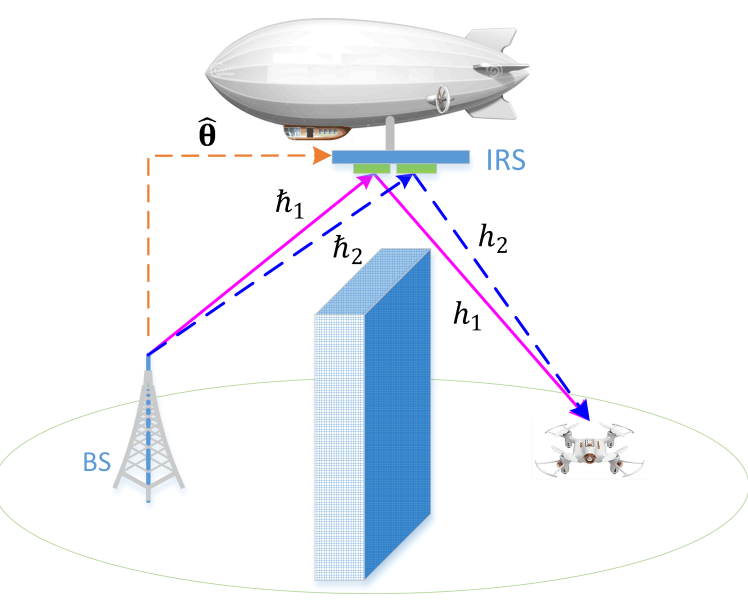

Fig. 1: IRS assisted UAV network.

their indices form the set $\mathbb{L}=\{1,2, \ldots, L\}$. This work considers that no direct link exists between the BS and LAUAV due to large obstacles. Therefore, the signal is transmitted from the BS to the LAUAV with the assistance of an IRS panel attached to HAP. The reflecting elements of the IRS panel introduce phase shifts to the signals of the BS and reflect them to the LAUAV. The passband BS signal arriving at the $i$ th IRS reflecting element can be expressed as

$$
r_{i}(t)=a h_{i} \cos \left(2 \pi f_{\mathrm{c}} t+\varphi-\psi_{i}\right), \quad i \in \mathbb{L}
$$

where $a$ is the amplitude and $\varphi$ is the phase of the transmitted information symbol, $f_{\mathrm{c}}$ is the carrier frequency, $h_{i}$ is the channel gain from the BS to the $i$ th reflecting element, and $\psi_{i}$ is the phase shift introduced by the channel. The signal reflected by each IRS element is attenuated by a factor $g_{i}$ and its phase is shifted by $\theta_{i}$. The signal received at the destination LAUAV from the $L$ reflecting elements of the HAP is given as

$$
y(t)=\sum_{i=1}^{L} \hbar_{i} h_{i} g_{i} a \cos \left(2 \pi f_{\mathrm{c}} t+\varphi-\psi_{i}-\phi_{i}+\theta_{i}\right)+n(t)
$$

where $n(t)$ is $\mathcal{N} \sim\left(0, \sigma_{\mathrm{n}}^{2}\right), \hbar_{i}$ and $\phi_{i}$ are respectively the gain and phase of IRS to LAUAV channel. The values of $\theta_{i} \forall i$ should be selected such that the phases of all reflected signals are aligned at the receiver, i.e., $\theta_{i}=\psi_{i}+\phi_{i}$. However, the estimated cascaded channel phase $\hat{\theta}_{i}$ can not be estimated and compensated perfectly, which leads to phase alignment errors that can be defined as $\epsilon_{i} \triangleq$ $\theta_{i}-\hat{\theta}_{i}, \epsilon_{i} \in[-\pi, \pi)$. The received signal with imperfect phase compensation can be expressed as

$$
y(t)=a \sum_{i=1}^{L} A_{i} \cos \left(2 \pi f_{\mathrm{c}} t+\varphi+\epsilon_{i}\right)+n(t)
$$

where $A_{i}=\hbar_{i} h_{i} g_{i} \in(-\infty, \infty)$. Without loss of generality we assume that $g_{i}=1$, and thus, the cascaded channel $\hbar_{i} h_{i} \triangleq A_{i}$. After downconversion to baseband, and considering the sinusoidal addition theorem [11], the received signal inphase and quadrature components can be written as $y_{I}=B_{L} \cos \left(\varphi+\epsilon_{i}\right)+n_{I}$ and $y_{Q}=$ $B_{L} \sin \left(\varphi+\epsilon_{i}\right)+n_{Q}$. For phase shift keying (PSK) modulation, the information symbol amplitude $a=1$.

In UAV communications, ground-to-air and air-to-air channels, $\hbar_{i}$ and $h_{i}$, typically have a dominant LoS component, and thus can be modelled using the Rician distribution with a large $K$ factor [12]. It was experimentally found that the Rician factor for air-toair and ground-to-air can be about $12 \mathrm{~dB}$ for C-band and L-band signalling [13], [14]. Consequently, free space pathloss dominates the channel gain $A_{i}$ [3], [4]. Moreover, the transmitted signals experience spatially distributed atmospheric distortions caused by the local temperature variations. Therefore, the channels' gains between the BS and each IRS element may experience different attenuation such that $h_{i} \neq h_{j}, \forall i \neq j,\{i, j\} \in \mathbb{L}$. The same argument also applies to IRS-LAUAV channels [15].

Channel estimation in IRS can be performed by dividing the coherence time interval $T$ into two time slots $T_{1}$ and $T_{2}$. The time interval $T_{1} \ll T_{2}$ is allocated for phase estimation, where $\hat{\theta}_{i} \forall i$ is estimated and fed to the corresponding IRS element controller. After phase estimation and compensation, the data transmission is performed in $T_{2}$. For channel estimation, we consider the protocol proposed in [16]-[18]. Therefore, $T_{1}$ is divided into $L$ sub-slots each of which has a duration of $T_{1} / L$, where only one reflector is switched on in each sub-slot while all other IRS elements are switched off. Therefore, the received complex baseband signal during the channel estimation of the $l$ th IRS element is $y_{l}=$ $A_{l} s_{l} \exp (j \theta)+n$, where $s_{l}$ is pilot symbol. For least square estimation (LSE), the channel can be estimated as $\hat{H}_{l}=y_{l} / s_{l}$, and thus, $\hat{\theta}_{i}=\arctan \left(\frac{\hat{H}_{Q, l}}{\hat{H}_{I, l}}\right)$ where $\hat{H}_{I}$ and $\hat{H}_{Q}$ are the inphase and quadrature components of $\hat{H}_{l}$. In such scenarios, the probability distribution function (PDF) of the phase error $\epsilon_{l}$ is given by

$f(\epsilon)=\frac{\mathrm{e}^{-\gamma \beta^{2}}}{2 \pi}\left[1+\sqrt{\gamma \pi} \beta \cos (\epsilon) \mathrm{e}^{\gamma \beta^{2} \cos ^{2}(\epsilon)} \operatorname{erfc}(-\sqrt{\gamma} \beta \cos (\epsilon))\right]$

where $\gamma, \beta$ as well as the derivation of (4) are given in Appendix A and $\operatorname{erfc}(x)=\frac{2}{\sqrt{\pi}} \int_{0}^{\infty} \mathrm{e}^{-t^{2}} d t$. As can be noted from (4), the PDF of phase error is complicated and using it for further analysis would mostly yield intractable solutions. Therefore, as depicted in Appendices B and C, $f(\epsilon)$ can be closely approximated by the von Mises PDF [4], [9] for a wide range of SNRs. Therefore, we consider that

$$
f_{\epsilon}\left(\epsilon_{i}\right)=\frac{1}{2 \pi I_{0}\left(\kappa_{i}\right)} e^{\kappa_{i} \cos \left(\epsilon_{i}-\mu_{i}\right)}
$$

where $I_{0}(\cdot)$ is the modified Bessel function of the first kind and zero order, $\mu_{i}$ is the mean, $\mu_{i}=0$ for unbiased estimators, and $\kappa_{i}$ is the concentration parameter of the PDF, which captures the phase estimation accuracy. The value of $\kappa$ is generally proportional to SNR, and thus when $\kappa$ increases the phase estimation quality improves. Ultimately, $\kappa \rightarrow \infty$ when $S N R \rightarrow \infty$.

\section{BER AWARE IRS ELEMENT SELECTION}

\section{A. BER Analysis}

To simplify the analysis, we consider binary phase shift keying (BPSK) modulation. Moreover, to generalize the problem, we consider the case that the LAUAV can switch off the $l$ th IRS element by setting $g_{l}=0$. Therefore, the BER from BS to LAUAV with $L$ reflectors is given as [3]

$$
P_{E}(\boldsymbol{\alpha})=\frac{1}{\sqrt{2 \pi \sigma_{Y_{L}}^{2}}} \int_{B_{L, m}^{2}}^{B_{L, M}^{2}} Q(X) \mathrm{e}^{-\frac{\left(y_{L}-\mu_{Y_{L}}\right)^{2}}{2 \sigma_{Y_{L}}^{2}}} d y_{L}
$$




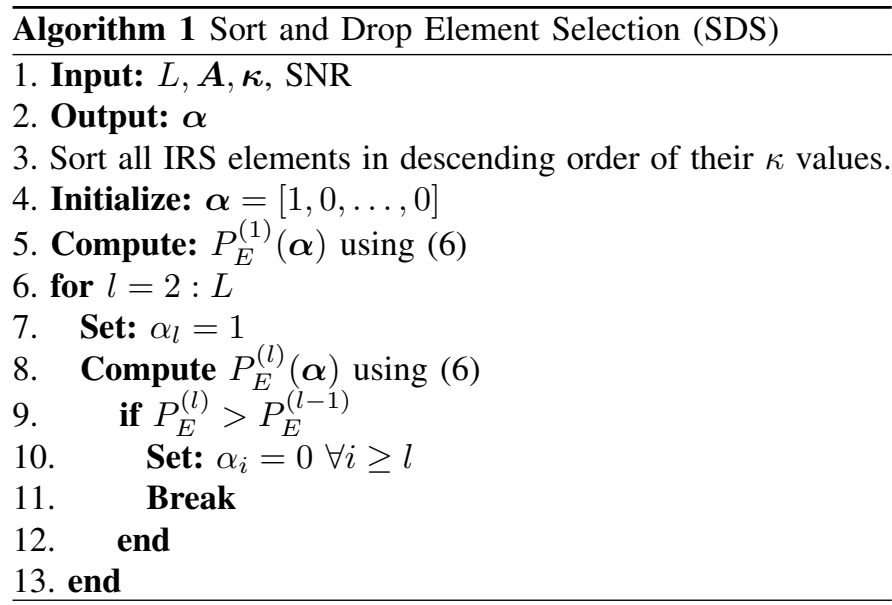

where $\boldsymbol{\alpha}=\left[\alpha_{1}, \alpha_{2}, \ldots, \alpha_{L}\right], \alpha_{l} \in\{0,1\} \forall l, Q(X)=$ $1 / 2 \operatorname{erfc}(X / \sqrt{2}), X=\sqrt{2 y_{L} / \sigma_{Z}^{2}}, Y_{L}$ is the signal envelope which can approximated as a Gaussian distribution using central limit theorem (CLT) for large $L, \mu_{Y_{L}}$ and $\sigma_{Y_{L}}^{2}$ are the mean and variance of $Y_{L}$, respectively. The signal envelope is $Y_{L} \triangleq B_{L}^{2} \in$ $\left[B_{L, m}^{2}, B_{L, M}^{2}\right]$, where $B_{L, m}^{2}=0$ and $B_{L, M}^{2}=\sum_{i=1}^{L} \alpha_{i} A_{i}$. The mean $\mu_{Y_{L}}$ and variance $\sigma_{Y_{L}}^{2}$ can be represented as [3]

$$
\mu_{y_{L}}=\sum_{i=1}^{L} \alpha_{i} A_{i}^{2}+2 \sum_{j=2}^{L} \sum_{k=1}^{j-1} \alpha_{j} \alpha_{k} A_{j} A_{k} \frac{I_{1}\left(\kappa_{j}\right) I_{1}\left(\kappa_{k}\right)}{I_{0}\left(\kappa_{j}\right) I_{0}\left(\kappa_{k}\right)}
$$

and

$$
\sigma_{Y_{L}}^{2}=\mathbb{E}\left[Y_{L}^{2}\right]-\mu_{Y_{L}}
$$

where $\mathbb{E}[\cdot]$ is the expectation operation, and $\mathbb{E}\left[Y_{L}^{2}\right]$ is given by

$$
\begin{aligned}
\mathbb{E}\left[Y_{L}^{2}\right]= & \left(\sum_{i=1}^{L} \alpha_{i} A_{i}\right)^{4}+4|A| \sum_{j=2}^{L} \sum_{k=1}^{j-1} \alpha_{j} \alpha_{k} \mathbb{E}\left[\cos \left(\phi_{j k}\right)\right] \\
& +4 \mathbb{E}\left[\left(\sum_{j=2}^{L} \sum_{k=1}^{j-1} \alpha_{j} \alpha_{k} A_{j} A_{k} \cos \left(\phi_{j k}\right)\right)^{2}\right]
\end{aligned}
$$

where $\phi_{j k}=\phi_{j}-\phi_{k}$.

Based on the BER in (6), it can be numerically demonstrated that $P_{E}$ is not strictly decreasing versus $L$ when the values of $\kappa_{i}$ are not equal for all values of $i$. Consequently, only a subset of elements should be selected while all others should be switched off.

\section{B. IRS Element Selection}

To minimize $P_{E}$ by selecting a certain subset of IRS elements, the values of $\boldsymbol{\alpha}$ should be selected such that

$$
\arg \min _{\boldsymbol{\alpha}} P_{E}(\boldsymbol{\alpha}) \text {. }
$$

However, the optimization problem in $(10)$ is an NP-hard binary optimization problem with a non-linear objective function, and thus it cannot be solved in polynomial time. Therefore, we propose a heuristic algorithm to solve the formulated problem in (10).

Generally speaking, increasing the number of IRS elements $L$ improves the BER. However, when some elements have large phase errors, then the BER might degrade by using such elements. The impact of including a certain IRS element depends on several

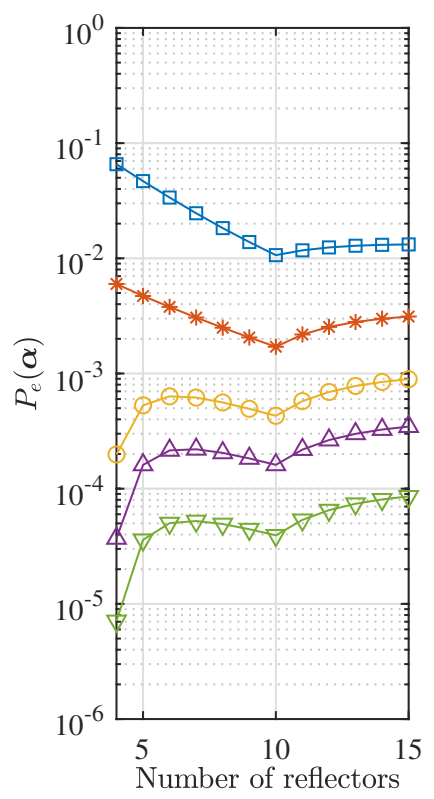

a) $\kappa_{1}$

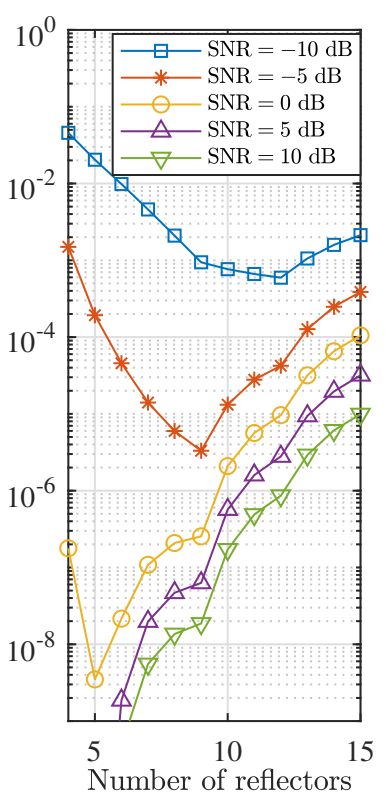

b) $\kappa_{2}$
Fig. 2: Relationship between number of selected reflectors and BER for $L=15$.

parameters such as the phase error of that element as well as the other used elements, SNR and $L$. Therefore, the general trend is that there is a threshold for the phase error such that any element with phase error larger than the threshold should be excluded. For example, Fig. 2 shows the BER for two different sets of $\kappa$ values, $\boldsymbol{\kappa}_{1}=[20,20,20,1,1,1,1,1,1,1,0,0,0,0,0]$ and $\boldsymbol{\kappa}_{2}=[20,20,20,3,3,2,2,2,2,1,1,1,0,0,0]$. As can be noted from the figure, the optimum number of elements depends on SNR as well as the value of $\kappa$ for the other reflectors. Consequently, we propose the SDS algorithm as a low complexity alternative for the optimum solution. The SDS initially sorts all IRS elements based on their phase error statistics, which is indicated by $\kappa$, and then tests the impact of including each IRS element on the BER. The algorithm stops when adding an element incresaes the BER. Algorithm 1 summarizes the proposed SDS algorithm.

As described in Algorithm 1, all reflectors are sorted in a descending order based on their $\kappa$ values. Then the first element is selected from the sorted set and $P_{E}([1,0,0, \ldots, 0])$ is computed. In the second iteration, the second element in the sorted set is included and $P_{E}([1,1,0, \ldots, 0])$ is computed. Then, $P_{E}$ in the second iteration $P_{E}^{(2)}$ is compared with $P_{E}$ that was obtained in the first iteration $P_{E}^{(1)}$. If $P_{E}^{(2)}<P_{E}^{(1)}$ the algorithm proceeds to the third iteration where $\boldsymbol{\alpha}=[1,1,1,0,0 \ldots, 0]$. If $P_{E}^{(3)}<P_{E}^{(2)}$ then the algorithm starts another iteration, otherwise it stops, and so forth. Although the SDS algorithm may not give the optimum solution, it offers a reasonable BER performance with low-complexity and less number of reflectors.

It should be noted that $(6)$ is generally accurate for large values of $L$, roughy $L>3$, because it is based on the CLT. Therefore, Algorithm 1 should start at $l=4$. In the case that a small number of reflectors should be considered, then Algorithm 1 remains unchanged, except that the BER for $l<4$ should be computed as reported in [3]. 
TABLE I: The BER and total number of reflectors selected by SDS algorithm for $L=20$.

\begin{tabular}{|c|c|c|c|c|c|c|c|c|c|c|c|c|}
\hline SNR & \multicolumn{4}{|c|}{$-20 \mathrm{~dB}$} & \multicolumn{4}{|c|}{$-6 \mathrm{~dB}$} & \multicolumn{4}{|c|}{$-2 \mathrm{~dB}$} \\
\hline$\kappa_{i} \forall i$ & 0 & 1 & 2 & 20 & 0 & 1 & 2 & 20 & 0 & 1 & 2 & 20 \\
\hline$P_{e}(\boldsymbol{\alpha})$ & 0.17 & $5.5 \cdot 10^{-2}$ & $1.6 \cdot 10^{-2}$ & $2.9 \cdot 10^{-3}$ & $2.6 \cdot 10^{-4}$ & $2.2 \cdot 10^{-4}$ & $1.6 \cdot 10^{-8}$ & 0 & $2 \cdot 10^{-8}$ & $2 \cdot 10^{-8}$ & $3.7 \cdot 10^{-9}$ & 0 \\
\hline$L_{S D S}$ & 20 & 20 & 20 & 20 & 5 & 6 & 20 & 20 & 5 & 5 & 7 & 20 \\
\hline
\end{tabular}

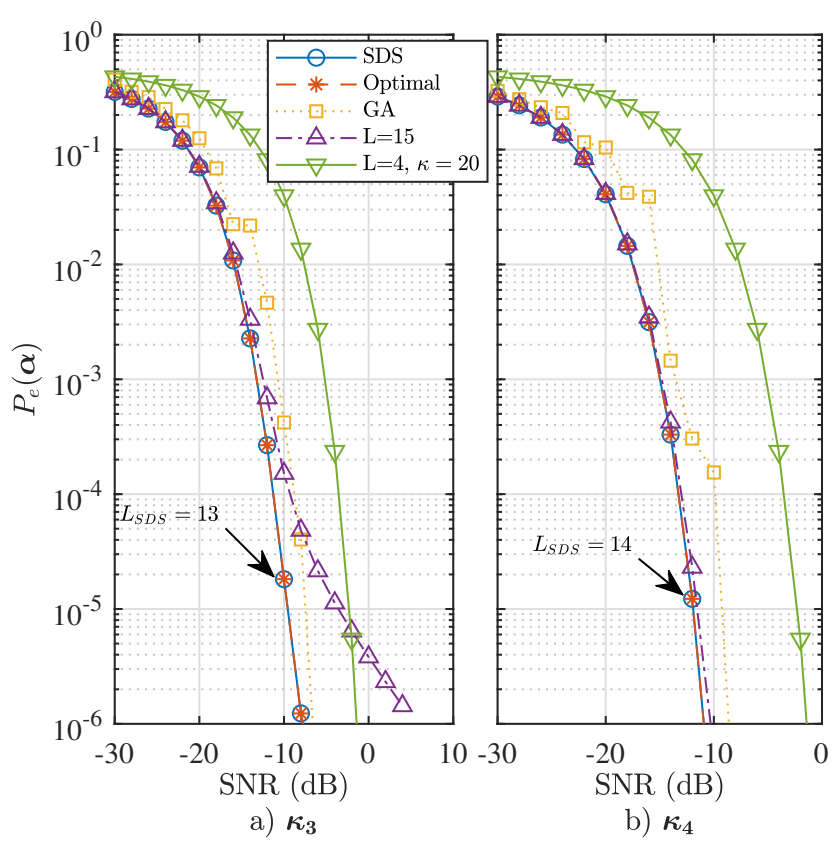

Fig. 3: BER of the SDS algorithm in comparison to other selection schemes using $L=15$.

\section{PERformance Evaluation}

This section presents the performance of the proposed SDS algorithm and compares it to the optimum solution as well as to heuristic GA. The optimum solution uses exhaustive search over $L$ ! combinations. In the heuristic GA, the BER is used as the fitness function, which is computed for each selected individual set of reflectors, and the next population is based on the current generation with the best fitness.

Table $\llbracket$ shows the SDS BER and selected number of reflectors where where all reflectors have the same value of $\kappa$. The table shows that the total number of selected elements $L_{S D S}$ is generally inversely proportional to SNR and directly proportional to $\kappa$. Such performance is obtained because the BER at low SNRs is dominated by the additive white Gaussian noise (AWGN), and hence, increasing $L_{S D S}$ reduces BER. When the SNR is increased to $-6 \mathrm{~dB}, L_{S D S}$ increases with the increase of $\kappa$. In this case, the BER depends on both the phase noise and SNR. Therefore, $L_{S D S}$ increases when the phase error decreases.

Fig. 3 compares the BER using SDS, the optimum solution, and GA for $L=15$. Moreover, the BER is presented when all reflectors are used, and when the best 4 reflectors are selected. The used sets of $\kappa$ are, $\kappa_{3}$ with 4 reflectors have $\kappa=20$, while $\kappa$ for the remaining reflectors is uniformly distributed over $[0,1]$. On the other hand, $\boldsymbol{\kappa}_{\mathbf{4}}=[20,20,20,20,20,9,8,7,6,5,4,3,2,1,0]$. Both Fig. 33 and Fig. 33 show that the SDS algorithm perfectly matches the optimum. Fig. 37 shows that a noticeable improvement is obtained by the SDS algorithm when compared to the case of $L=15$ for SNR $>-10 \mathrm{~dB}$. Fig. 3p shows that that the BER using the SDS algorithm provides comparable BER to the all reflectors selection scenario over the considered SNR range. This behaviour confirms the fact that the selection of a few reflectors with accurate phase compensation can provide equal or lower BER. It can be seen also that the performance of GA approaches the other algorithms at certain SNR values, while it deviates at other SNR values. Interestingly, the selection of the best 4 reflectors provides worse BER compared to SDS, GA, and the optimal solution.

\section{Conclusion}

In this letter, a reflectors selection algorithm for UAV-IRS network was introduced for imperfect phase estimation and compensation scenario with the aim of minimizing the BER. Additionally, the distribution of the phase error is derived in a closed form, and an accurate approximation was provided using von Mises PDF with parameter $\kappa$. The selection depends on the SNR and the $\kappa$ value of each reflector. The obtained BER results show that the proposed SDS algorithm provides BER enhancement as compared to all-reflectors selection, and a comparable performance with the optimal algorithm.

\section{APPENDIX}

\section{A. Phase Error Distribution}

As discussed in Sec. III the channel gain is dominated by a LoS component. For a deterministic complex channel, the channel can be represented as $H=H_{I}+j H_{Q} \triangleq \beta \mathrm{e}^{j \theta}$. To estimate the channel phase, a pilot symbol $s$ is transmitted, and the received signal is given by

$$
y=H s+w
$$

where $w \sim \mathcal{C N}\left(0,2 \sigma_{\mathrm{w}}^{2}\right)$ is the AWGN. Given that $s=1$, then $y=\left(H_{I}+w_{I}\right)+j\left(H_{Q}+w_{Q}\right)$. Consequently, the phase estimate is obtained as $\hat{\theta}=\arctan \left(\frac{H_{Q}+w_{Q}}{H_{I}+w_{I}}\right)$. Due to the presence of the AWGN, there will be a phase error $\epsilon=\hat{\theta}-\theta$. By noting that $y_{I}$ and $y_{Q}$ are independent and identically distributed Gaussian random variables, $y_{I} \sim \mathcal{N}\left(H_{I}, \sigma_{w}^{2}\right)$ and $y_{Q} \sim \mathcal{N}\left(H_{Q}, \sigma_{w}^{2}\right)$, then their joint PDF is given by,

$$
f\left(y_{I}, y_{Q}\right)=\frac{1}{2 \pi \sigma_{w}^{2}} \mathrm{e}^{\frac{-1}{2 \sigma_{w}^{2}}\left(y_{I}^{2}+y_{Q}^{2}+\beta^{2}-2\left(y_{I} H_{I}+y_{Q} H_{Q}\right)\right)} .
$$

The PDF of $\hat{\theta}$ can be obtained by converting the PDF to polar coordinates $y_{I}=r \cos \hat{\theta}$ and $y_{Q}=r \sin \hat{\theta}$ to obtain $f(r, \hat{\theta})$, and then averaging over $r$, consequently, we obtain

$$
f_{\hat{\Theta}}(\hat{\theta})=\frac{\gamma \mathrm{e}^{-\gamma \beta^{2}}}{\pi} \int_{0}^{\infty} r \mathrm{e}^{-\gamma\left(r^{2}-2 r\left(H_{I} \cos \hat{\theta}+H_{Q} \sin \hat{\theta}\right)\right)} d r
$$

where $\gamma=1 /\left(2 \sigma_{\mathrm{w}}^{2}\right)$. Using [19, (2.3.15.7), pp 344] and after some manipulations we obtain,

$$
f_{\hat{\Theta}}(\hat{\theta})=\frac{e^{-\gamma \beta^{2}}}{2 \pi}\left(1+\sqrt{\gamma \pi} z(\hat{\theta}) \mathrm{e}^{\gamma z^{2}(\hat{\theta})} \operatorname{erfc}(-\sqrt{\gamma} z(\hat{\theta}))\right)
$$


where $z(\hat{\theta})=H_{I} \cos \hat{\theta}+H_{Q} \sin \hat{\theta}$. Finally, since $\epsilon=\hat{\theta}-\theta$, the phase error distribution $f_{\epsilon}(\epsilon)=f_{\hat{\Theta}}(\epsilon+\theta)$ can be expressed as (14), except that $z(\hat{\theta})$ is replaced by $\tilde{z}(\theta)$, where $\tilde{z}(\theta)=H_{I} \cos (\epsilon+\theta)+H_{Q} \sin (\epsilon+\theta)$ which can be written as $\tilde{z}(\theta)=\beta \cos (\epsilon)$. For ease of notation, we use $f_{\epsilon}(\epsilon)$ as $f(\epsilon)$. Consequently, $f(\epsilon)$ can be expressed as (4).

\section{B. High SNR Scenario}

It can be noted that at high SNRs, the second term inside the brackets in (14) is $\gg 1, \beta$ can be normalized to 1 and $\operatorname{erfc}($.$) can$ be approximated as

$$
\operatorname{erfc}(-\sqrt{\gamma} \cos (\epsilon))=2 \Phi(\epsilon)
$$

where $\Phi(\epsilon)=u\left(\epsilon+\frac{\pi}{2}\right)-u\left(\epsilon-\frac{\pi}{2}\right)$ and $u(\cdot)$ is the Heaviside unit step function. Consequently,

$$
f(\epsilon) \approx \sqrt{\frac{\gamma}{\pi}} \Phi(\epsilon) \mathrm{e}^{-\gamma\left(1-\cos ^{2}(\epsilon)\right)+\ln \cos (\epsilon)} .
$$

It can noted that $-\gamma\left(1-\cos ^{2}(\epsilon)\right)$ and $\ln (\cos (\epsilon))$ are both negative valued functions for $\epsilon \in\left(\frac{-\pi}{2}, \frac{\pi}{2}\right)$ and $-\gamma\left(1-\cos ^{2}(\epsilon)\right) \ll$ $\ln (\cos (\theta))$ at high SNRs. Moreover, by applying the trigonometric identity $\cos (2 \epsilon)=2 \cos ^{2}(\epsilon)-1, f(\epsilon)$ can be approximated as

$$
f(\epsilon) \approx \frac{\Phi(\epsilon)}{2 \pi c} \mathrm{e}^{\frac{\gamma}{2} \cos (2 \epsilon)}
$$

where $c=\frac{\mathrm{e}^{\frac{\gamma}{2}}}{2 \sqrt{\gamma \pi}}$. By defining $\kappa \triangleq \frac{\gamma}{2}$ and using the modified Bessel function approximation [20], we obtain

$$
I_{0}(\kappa) \approx \frac{1}{\sqrt{\gamma \pi}} \mathrm{e}^{\frac{\gamma}{2}} \triangleq 2 c .
$$

Consequently, $f(\epsilon)$ can be written as

$$
f(\epsilon) \approx \frac{2 \Phi(\epsilon)}{2 \pi I_{0}(\kappa)} \mathrm{e}^{\kappa \cos (2 \epsilon)}
$$

\section{Phase Error Estimation Approximation to von Mises Distribution}

To be able to approximate $f(\epsilon)$ as the standard form of von Mises distribution, we equate $f(\epsilon)$ to the standard form of von Mises given in 5. Thereafter, the same approximation used above for $I_{0}(\cdot)$ can be applied to obtain

$$
2 \sqrt{\kappa} \mathrm{e}^{\kappa(\cos (2 \epsilon)-1)}=\sqrt{\hat{\kappa}} \mathrm{e}^{\hat{\kappa}(\cos (\epsilon)-1) .}
$$

Applying the trigonometric identity $\cos (2 \epsilon)=2 \cos ^{2}(\epsilon)-1$, and then the difference between two squares rule yields

$$
2 \sqrt{\kappa} \mathrm{e}^{(\cos (\epsilon)-1)(2 \kappa(\cos (\epsilon)+1)-\hat{\kappa})}=\sqrt{\hat{\kappa}} .
$$

Since we aim at equating the two functions in (21) for any value of $\epsilon$, it can be observed that for any value of $\epsilon \in\left(\frac{-\pi}{2}, \frac{\pi}{2}\right)$, the equality is satisfied if and only if $4 \kappa=\kappa$. Finally, by setting $\kappa=4 \kappa=2 \gamma, f(\epsilon)$ can be written in von Mises form as

$$
f(\epsilon) \approx \frac{\Phi(\epsilon)}{2 \pi I_{0}(\hat{\kappa})} \mathrm{e}^{\hat{\kappa} \cos (\epsilon)} .
$$

\section{REFERENCES}

[1] C. Liaskos et al., "A new wireless communication paradigm through software-controlled metasurfaces," IEEE Commun. Mag., vol. 56, no. 9, pp. 162-169, 2018.

[2] M. A. Al-Jarrah et al., "Performance analysis of wireless mesh backhauling using intelligent reflecting surfaces," IEEE Trans. Wireless Commun., vol. 20 , no. 6, pp. 3597-3610, 2021.

[3] M. Al-Jarrah et al., "On the performance of IRS-assisted multilayer UAV communications with imperfect phase compensation," to appear in IEEE Trans. Commun., 2021. [Online]. Available: https: //doi.org/10.36227/techrxiv.13153211.v1.

[4] M. Al-Jarrah, E. Alsusa, A. Al-Dweik, and D. K. C. So, "Capacity analysis of IRS-based UAV communications with imperfect phase compensation," IEEE Wireless Commun. Lett., vol. 10, no. 7, pp. 1479-1483, 2021.

[5] K. Zhi, C. Pan, H. Ren, and K. Wang, "Uplink achievable rate of intelligent reflecting surface-aided millimeter-wave communications with low-resolution ADC and phase noise," IEEE Wireless Commun. Lett., vol. 10, no. 3, pp. 654-658, 2021.

[6] S. Gong, Z. Yang, C. Xing, J. An, and L. Hanzo, "Beamforming optimization for intelligent reflecting surface-aided SWIPT IoT networks relying on discrete phase shifts," IEEE Internet Things J., vol. 8, no. 10, pp. 8585-8602, 2021.

[7] V. Jamali, M. Najafi, R. Schober, and H. V. Poor, "Power efficiency, overhead, and complexity tradeoff of IRS codebook design-Quadratic phase-shift profile," IEEE Wireless Commun. Lett., vol. 25, no. 6, pp. 2048-2052, 2021.

[8] M.-M. Zhao, Q. Wu, M.-J. Zhao, and R. Zhang, "IRS-aided wireless communication with imperfect CSI: Is amplitude control helpful or not?" in GLOBECOM, 2020, pp. 1-6.

[9] R. Ferreira et al., "Bit error probability for large intelligent surfaces under double-Nakagami fading channels," IEEE Open J. Commun. Soc., vol. 1, pp. 750-759, 2020.

[10] S. Wang and B. Meng, "Resource allocation and scheduling problem based on genetic algorithm and ant colony optimization," in PAKDD. Berlin, Heidelberg: Springer-Verlag, 2007, p. 879-886.

[11] Y. Maghsoodi and A. Al-Dweik, "Error-rate analysis of FHSS networks using exact envelope characteristic functions of sums of stochastic signals," IEEE Trans. Veh. Technol., vol. 57, no. 2, pp. 974-985, 2008.

[12] W. Khawaja, I. Guvenc, D. W. Matolak, U.-C. Fiebig, and N. Schneckenburger, "A survey of air-to-ground propagation channel modeling for unmanned aerial vehicles," IEEE Commun. Surveys Tuts., vol. 21, no. 3, pp. 2361-2391, 2019.

[13] D. W. Matolak and R. Sun, "Air-ground channel characterization for unmanned aircraft systems - part i: Methods, measurements, and models for over-water settings," IEEE Trans. Veh. Technol., vol. 66, no. 1, pp. 26-44, 2017.

[14] _ "Air-ground channel characterization for unmanned aircraft systems-part iii: The suburban and near-urban environments," IEEE Trans. Veh. Technol., vol. 66, no. 8, pp. 6607-6618, 2017.

[15] L. Cang, H.-K. Zhao, and G.-X. Zheng, "The impact of atmospheric turbulence on Terahertz communication," IEEE Access, vol. 7, pp. 88 685-88 692, 2019.

[16] T. L. Jensen and E. De Carvalho, "An optimal channel estimation scheme for intelligent reflecting surfaces based on a minimum variance unbiased estimator," in ICASSP, 2020, pp. 5000-5004.

[17] D. Mishra and H. Johansson, "Channel estimation and low-complexity beamforming design for passive intelligent surface assisted MISO wireless energy transfer," in ICASSP, 2019, pp. 4659-4663.

[18] P. Wang, J. Fang, X. Yuan, Z. Chen, and H. Li, "Intelligent reflecting surface-assisted millimeter wave communications: Joint active and passive precoding design," IEEE Trans. Veh. Technol., vol. 69, no. 12, pp. 14960-14973, 2020.

[19] A. Prudnikov, Y. Brychkov, and O. Marichev, "Integrals and Series: Elementary Functions," Gordon and Breach Science Publishers, vol. 3rd edition, 1986.

[20] M. Abramowitz and I. Stegun, "Handbook of Mathematical Functions with Formulas," New York, vol. 3rd edition, 1972. 